\title{
À propos du pouvoir sacré en Afrique : que disent
} les textes?

On Sacred Power: What Do the Texts Say?

\section{Claude Tardits}

\section{(2) OpenEdition \\ Journals}

Édition électronique

URL : https://journals.openedition.org/span/855

DOI : $10.4000 /$ span. 855

ISSN : 2268-1558

Éditeur

École pratique des hautes études. Sciences humaines

\section{Édition imprimée}

Date de publication : 1 décembre 1990

Pagination : $35-48$

ISSN : 0294-7080

\section{Référence électronique}

Claude Tardits, «À propos du pouvoir sacré en Afrique : que disent les textes ? », Systèmes de pensée en Afrique noire [En ligne], 10 | 1990, mis en ligne le 17 juin 2013, consulté le 21 septembre 2021. URL: http://journals.openedition.org/span/855 ; DOI : https://doi.org/10.4000/span.855 


\title{
A PROPOS DU POUVOIR SACRE EN AFRIQUE :
}

\author{
QUE DISENT LES TEXTES ?
}

par

\section{Claude Tardits}

En guise d'introduction, je désirerais présenter des observations sur quelques points auxquels la lecture de l'invitation à la journée d'études sur les structures symboliques du pouvoir sacré en Afrique noire, organisée par L. de Heusch et M. Cartry le 27 janvier 1988, m'a conduit à prêter attention: je dirai d'abord quelques mots de Marc Bloch, ensuite plus longuement de Frazer et du thème du roi divin, enfin je parlerai des interrogations qui se sont posées à moi sur la sacralisation du pouvoir en Afrique.

C'est par son invitation au comparatisme que $M$. Bloch a retenu l'attention des anthropologues, en particulier à la suite de ses propositions typologiques sur la société féodale (Bloch, 1949: 252). La société féodale, pour Bloch, n'était pas un phénomène strictement européen et la recherche d'une féodalité africaine a, nous nous en souvenons, alimenté plusieurs débats chez les africanistes. La référence à la féodalité apparait dans des textes touchant des populations aussi différentes que les Nzakara, les Bariba, les Nyoro, les Rwandais, les Mossi et les Nupe. Quelques mises au point intervinrent ultérieurement, auxquelles contribuèrent historiens et anthropologues, qui 
entraînèrent la disparition de l'Afrique féodale (Chilver, in Richards, 1959: 378-393; Goody, 1971: 1-20).

Par contre, pas ou peu de références aux Rois thaumaturges (1924, 2è ed. 1983) dans les ouvrages sur les royautés d'Afrique. Pourtant ici Bloch nous invite directement à la réflexion puisqu'il mentionne dans son texte non seulement les chefs mélanésiens mais les chefs africains: il nous renvoie en particulier aux petits souverains sénégalais du Wallo qui étaient censés agir sur la nature. Les textes étaient sans doute fort vite lus car, si les monarques devaient faire en sorte que les terres soient fertiles, ils n'étaient pas en charge de la guérison des maladies (Labouret, 1941: 87). Il n'existe pas à ma connaissance de rois thaumaturges en Afrique qui puissent trouver place à côté des rois de France et d'Angleterre, seuls souverains d'Europe qui, pendant 800 à 900 ans d'histoire, ont touché les malades affligés d'écrouelles. L'affaire était importante puisque, par exemple en juin 1716, Louis XIV, proche de la mort, touche à la veille de la Pentecôte 1700 personnes (Bloch, 1983: 383). Au 18ème siècle on commence en Angleterre à tourner le pouvoir royal en dérision et Hume peut écrire que cet usage "était atteint de ridicule aux yeux de tous les hommes de bon sens". La pratique cesse en France en 1825 avec Charles X. Lorsqu'on s'efforce de comprendre cette pratique, il ne faut oublier la célèbre formule qui apparaît au 16ème siècle: "le roi te touche, Dieu te guérit". Elle a donné lieu à bien des débats où l'on soulignait, selon que l'on était du côté de l'orthodoxie chrétienne ou de la politique, le rôle instrumental du roi ou les vertus inhérentes au monarque. Il est toutefois évident que le roi n'est point divin, situation impossible en pays chrétien. En Afrique, la santé est l'affaire des ancêtres lignagers ou des ancêtres royaux en quelques cas d'épidémie. Et, ne l'oublions pas, Bloch conclut son magisral travail par cette réflexion: "Ainsi, il est difficile de voir dans la foi au miracle royal autre chose qu'une erreur collective" (Bloch, 1983: 429). Ce thème n'est pas ici des nôtres.

Frazer nous concerne plus directement parce que l'Afrique a, pendant une ou deux décennies, été tenue comme la terre privilégiée 
des rois divins. Rappelons d'abord deux aspects fondamentaux de la démarche frazérienne qui la rattache à l'évolutionnisme du 19ème siècle: d'abord Frazer distingue un "premier temps de la magie" et un "second temps de la religion"; ensuite il assimile l'homme à la divinité parce que précisément l'homme possède des pouvoirs surnaturels qui, dans sa pensée, étaient un attribut de la divinité.

Les quatre points de la thèse de Frazer sur les rois divins ont été présentés dans une définition ramassée et précise de Seligman dans la "Frazer Lecture" de 1933: 1) le roi divin détient un pouvoir sur la nature; 2) il est le centre dynamique de l'univers; 3) ses actes et le cours de son existence doivent être soigneusement ordonnés; 4) il doit être mis à mort ou invité à se suicider à l'approche de la sénéscence ou après un certain nombre d'années de règne (Seligman, 1934: 4-8). La pensée de Frazer est cohérente, en particulier en raison du lien entre les points 4 et 1 : le pouvoir sur la nature commandé par la santé et la force du roi. Aussi, lorsqu'elles risquent de décliner, le monarque doit-il être éliminé et remplacé afin que soit sauvegardée la communauté politique.

Pendant les années 30 , quelques africanistes, presque tous attachés aux thèses diffusionnistes, ont cru pouvoir reconnaître parmi les souverains africains des rois divins. Seligman classe parmi ceuxci non seulement quelques chefs nilotiques dont le reth des Shilluk, mais aussi les monarques nyoro, ankolé, lovedu et shona à l'ouest; Meek, Jeffreys, Parrinder, Meyerowits qualifient de divins les monarques jukun, igala, umrundi, yoruba, edo et ashanti.

La réaction vient avec la critique que fit Evans-Pritchard de cette thèse à propos des Shilluk; puis la plupart des anthropologues anglais, attachés aux données de l'observation, écartèrent une expression qui ne tenait pas compte des réalités. Audrey Richards la rejeta pour les monarques des Etats interlacustres, Mair et Balandier l'ont ignoré dans leurs ouvrages généraux.

Ce qui s'est trouvé infirmé, ce sont les deux premières propositions de Frazer car il est bien établi que de nombreux interdits doivent être respectés par les souverains africains et leur entourage, et que plu- 
sieurs traditions font état d'élimination de souverains sénescents ou malades.

Revenons donc aux points 1 et 2 . Le souverain shilluk cherche bien à agir sur la nature, à obtenir la pluie, mais ceci comme les faiseurs de pluie dinka voisins, au moyen de sacrifices; le roi nyoro faisait des offrandes aux esprits ancestraux et à la divinité de l'abondance, Wamala, qui protège la population, les troupeaux et les récoltes, et il remettait, en cas de sécheresse, des animaux à sacrifier aux plus importants faiseurs de pluie. Aucune mention n'est faite, dans les textes de Roscoe, de propriétés propres au monarque, mais il fait état de recours qu'il revient au souverain d'exercer pour remplir son rôle. Il en est de même de la célèbre reine de la pluie lovedu: lorsqu'elle doit lutter contre la sécheresse et donc prévenir la famine, elle utilise d'une part des médecines conservées dans des pots de terre nourries de verdure et de termites, de l'autre, elle s'adresse aux ancêtres royaux, sacrifiant éventuellement sur les tombes de ces derniers. Les Krige ont ce commentaire: "Ce sacrifice est extrêmement rare, mais il illustre le mélange habituel et significatif du religieux avec ce qu'on devrait qualifier de rite purement magique" (Krige, 1936: 279).

Pour l'Afrique occidentale, indiquons que le roi jukun sacrifiait aussi à ses ancêtres et à diverses divinités (Meek, 1931: 266, 270, 293 ) et que les chefs yoruba propitiaient ancêtres et orisha spécialisés.

Tous les souverains africains, qu'ils aient été à une époque ou une autre qualifiés de divins ou non, ont une obligation, celle d'agir pour que la population et le bétail dont elle vit soient féconds, pour que les terres arrosées gardent leur fertilité et que soit écartée la famine. Ils remplissent ces obligations par des recours, directs ou indirects selon qu'ils sont eux-mêmes prêtres ou magiciens, à des actes magiques ou religieux. Dans les deux cas, les actes sont instrumentaux. L'échec, quel que soit l'âge du roi, peut entraîner son élimination. Citons, à titre d'exemple, cette information de Meek sur le roi jukun: "The king of the Jukun is, therefore, in virtue of his deity, able to control the rains and winds. A succession of droughts 
or bad harvests is ascribed to his negligence or to the waning of his strength, and he is accordingly secretly strangled" (Meek, 1931:130).

Une autre objection peut être faite à l'utilisation de l'expression roi divin; elle est fort différente mais de bonne méthode. C'est aux sociétés concernées, et ceci au niveau de leur langue, d'assimiler les souverains vivants aux ancêtres morts ou à ces entités religieuses qui se situent entre les ancêtres et la divinité créatrice; bref, dans un univers où le verbe est essentiel, le roi n'est pas dieu si la langue ne le dit pas et ici encore il faudrait, dans la façon dont on parle du roi, faire la part de la place laissée à l'hyperbole.

Est-ce à dire qu'il n'existe aucun cas de figure frazérienne dans les royautés africaines ? Eh bien si! Les activités sexuelles de citimukulu, le souverain des Bemba, stimulent la nature et Richards écrit à ce sujet: "C'est en raison de l'influence surnaturelle de ses actes sexuels que je trouve difficile de considérer le chef bemba simplement comme le grand prêtre d'un culte des ancêtres, ce qui autrement aurait été l'expression appropriée" (Richards, 1968: 29).

D'autres situations, que ne recouvrent pas les propositions de Frazer, nous renvoient toutefois à sa pensée: quel est, aux yeux de la population, le statut de ces souverains dans lesquels s'incarnent quelques composantes de la personne des rois défunts? Nous faisons référence au reth shilluk qui incarne Nyikang, le fondateur du royaume et à l'alafin d'Oyo qui représente Shango, l'un des grands souverains yoruba. Ces deux exemples, et il y en aurait beaucoup d'autres, conduisent à s'interroger à la fois sur le caractère de la relation entre le roi régnant et ses ancêtres, sur la signification, le caractère et les limites de ces identifications entre morts et vivants, sur ce qu'elles ajoutent au statut royal, et sur les différentes expressions de la successio universalis. Il y peut-être là matière à réflexion et à comparaison et certaines formes de mysticisme renverraient, comme l'a vu Monica Wilson, à la pensée frazérienne.

Le chef politique africain, qui n'est ni un thaumaturge ni une figure divine mais dont la singularité dans sa société est reconnue, s'est vu généralement qualifié de roi sacré. Le recours à cette expres- 
sion paraît fondé puisque, chef héréditaire, il acquiert son statut par un rituel qui le sépare de la population et lui confère la souveraineté, rituel quj s'accompagne parfois d'obligations aussi remarquables que celle de s'unir, une fois intronisé, par un acte réel ou symbolique à une soeur réelle ou classificatoire, voire à une femme assimilable à une soeur, situation que relève $M$. Izard chez les Mossi du Yatenga dans la description qu'il donne de l'intronisation du roi (Izard, 1985: 160); de plus, une fois intronisé, le nouveau roi est soumis à des règles qui commandent ses rapports avec la population, avec ses proches (serviteurs, épouses et parents), avec son corps et avec ses ancêtres; interdits qui, en le protégeant et en fixant, on pourrait écrire en figeant, une partie de ses comportements, sont la marque même de la sacralité. Code de conduite qui, en plusieurs cas, prévoit même sa mort, qualifiée, dans les commentaires qui en sont faits, tantôt de politique, tantôt de rituelle.

Un ensemble de questions me paraît ici se poser. La première, à laquelle la note de Luc de Heusch et de Michel Cartry fait référence, concerne les contextes politiques dans lesquels interviennent les rituels qui confèrent et sacralisent le pouvoir. Sont-ils propres au royaume ou les trouve-t-on au niveau des milliers de chefferies qui occupent la scène politique africaine ?

Je précise immédiatement que ce n'est pas ici, d'après moi, le lieu d'ouvrir un débat d'ordre typologique sur la distinction souvent faite entre chefferie et royaume. Remarquons simplement que les critères généralement retenus pour fonder une telle distinction semblent peu opératoires. La taille des unités politiques n'est pas un critère pertinent: il existe à travers le monde, hier comme aujourd'hui, des formations politiques dont les extensions territoriales et l'importance sont sans commune mesure et qui pourtant sont l'objet d'une même reconnaissance officielle. Quant aux différenciations sociales, critères dont on a souvent fait la marque de l'Etat, terme souvent utilisé pour classer les royaumes africains, il nous faut observer que les plus importantes d'entre elles, celles qui opposent la classe des hommes 
libres et la masse servile, se retrouvent dans des sociétés aux modes d'organisation les plus simples.

La confusion est, dans la pratique, certaine: pourquoi distinguer, au Cameroun, les chefferies bamiléké des royaumes tikar et bamoum alors qu'il s'agit de sociétés présentant les mêmes organisations politiques et les mêmes idéologies ? Pourquoi qualifier les unités politiques banyamwezi tantôt de royaume (Bösch, 1930: 493), tantôt de chefferie (Abrahams, 1967: 27) ? Quelque position que l'on prenne dans ce débat confus, on rencontre une sacralisation du pouvoir aussi bien dans ce qu'on appelle chefferie que royaume.

Pour justifier ces propos, on peut citer ces lignes de M. Dupire sur l'investiture des ardo des Peul nomades du Niger que l'on n'a jamais tenus pour des rois:

"L'ardo n'est que le conducteur d'un petit groupe de parents et par des contacts directs il doit pouvoir résoudre les problèmes qui se posent tant à l'intérieur de sa faction que dans ses rapports avec les populations voisines...

La nomination d'un chef s'accompagne d'une cérémonie qui s'appelle "le lavage de la chefferie" (lootingal laamu). Celle-ci qui était coutumière il $\mathrm{y}$ a une cinquantaine d'années a tendance à disparaître...

Rite de passage caractéristique, le lootingal laamu se compose d'une retraite de six jours encadrée de rites d'entrée et de sortie, d'un lavage du nouveau chef et de la présentation des hommages des membres de la fraction et des chefs voisins...

A la pleine lune, le 14 au matin, le chef fut conduit en cortège dans la grande case après s'être lavé seul la veille et sans témoins. Tandis que les ndottii'en rassemblés assistaient à son entrée, deux femmes l'accompagnèrent à l'intérieur. L'une, l'officiante qu'on appelle l'alkaali (juge), connaissait le rituel pour l'avoir pratiqué. Soutenant sous les aisselles le chef revêtu d'un boubou blanc et la tête couverte d'un pagne de couleur, elles marchaient lentement en accomplissant un pas de danse composé de petits piétinements. Elles l'aidèrent à s'asseoir sur un lit, sous lequel on avait étendu des feuilles de barkehi (barka = chance) et déposèrent à son côté une branche d'un arbre à fruits particulièrement prolifique...

Puis commença l'onction de henné. Sur chacune des mains de l'ardo l'alkaali versa de l'eau puis du henné, sept fois de suite, en prononçant le voeu "qu'Allah lui donne chance, longue vie et santé" et elle les entoura de feuilles épaisses de bambambi (calotropis procera) et de chiffons. Chacun des assistants reçut un peu de poudre de henné et l'oignon passa de main en main...

En procédant à l'application du henné et au lavage, l'officiante lançait les cris stridents des femmes "eh yo, lololo li.." et sur un ton chanté, lent et solennel, prononçait des paroles de félicita- 
tions, de souhait, aussi bien que de vulgaires commentaires des plus fantaisistes: "le voici notre chef, nous n'en avons jamais eu comme lui..." et après le départ d'un ancien chef venu le saluer: "il surpassera celui-là...". Sur le même ton grandiloquent, tout en serrant les bandelettes autour des mains elle poursuivait: "je ne veux pas entourer les mains de ces bandes sales..." puis dans un argot naï, manifestant son enthousiasme walla 'ardo banza (ce n'est pas un chef qui ne vaut rien) et enfin dans un style emphatique "heureuse suis-je, moi qui lave le fils d'un pélerin", c'est-à-dire un musulman...

Le septième jour de sa réclusion le chef fit sabrer un taureau de son troupeau dont la viande fut partagée entre tous les invités, selon les mêmes principes qui président aux distributions du worso. Mais une part symbolique - patte antérieure et testicules fut réservée au nouveau chef: c'est celle qui, chez les Wodaabe, est envoyée au père célébrant l'imposition du nom de son enfant...

$\mathrm{Au}$ début de cet après-midi commença le rite de sortie. Le chef quitta la cuuda accompagné de son garde du corps et de tout le cortège des visiteurs. Sur ses traces sa femme jetait un mélange de graines de haricots et de mil, préalablement humectées, image du rite des semailles qui est un symbole de fertilité de la Nature, à laquelle est associé le pouvoir du chef...

L'ardo ayant repris contact avec le monde extérieur, les réjouissances battirent leur plein: danses, chants, courses de chameaux." (Dupire, 1962: 291-294).

Le lecteur saura rapprocher ces lignes de M. Dupire des descriptions qui ont été données des intronisations royales et verra à quel point ces cérémonies sont similaires.

J'en viens maintenant à un point de méthode qu'aucune démarche critique ne saurait esquiver. Lorsque les anthropologues parlent de "roi sacré", formule de commodité certaine, peuvent-ils ignorer les expressions retenues par les sociétés af ricaines pour traduire l'image que ces sociétés se font de leurs chefs ? Les Peul font-ils référence à la figure de leur ardo dans des termes semblables à ceux qu'utilisent les Ashanti pour évoquer la figure de l'asentehene ? Par exemple, les rois bamoum doivent être "respectés" et le code qui les concerne s'appelle "le respect dû au roi". L'expression renvoie-t-elle vraiment à la notion de sacré ? Ajoutons que l'histoire de ce royaume connaît, au 19ème siècle, deux régicides qui ne suscitent, dans les textes bamoum, aucun commentaire sur le caractère sacrilège de tels actes.

Quelles que soient les données que l'on puisse être amené à comparer, il me paraît difficile de corréler les différentes expressions 
de la sacralité aux catégories d'une typologie politique incertaine. C'est ainsi que j'ai du mal à suivre Luc de Heusch lorsqu'il oppose un ordre symbolique de la royauté à un ordre symbolique lignager (Heusch, 1982: 25-26).

Venons-en, pour terminer, à la question à laquelle doit répondre toute démarche comparatiste, en particulier si elle relève d'une approche structuraliste. Quelles sont les données embrassées par l'expression "pouvoir sacré" qui sont susceptibles de s'ordonner en série et qui, relevant d'un système de transformation, s'éclairent les unes par les autres?

Peut-on comparer les rites d'intronisation? Disons tout de suite que la vingtaine de descriptions disponibles reposent presque totalement sur des déclarations de témoins et non sur des observations; l'information est de plus lacunaire sur les phases du rituel qui se déroule pendant les retraites ou les réclusions.

Les comparaisons permettent certes de dégager des similitudes formelles entre des séquences d'intronisation qui sont caractéristiques des rites de passage, mais bien des phases des intronisations symbolisent des événements historiques propres à la société concernée. Ces projections symboliques d'une histoire particulière ne relèvent d'aucun système. Peut-on vraiment parler de structures symboliques dans la mesure où une structure renvoie à des agencements qui forment système, ceci par définition ?

Peut-on plus facilement comparer les codes, les règles qui gouvernent la vie du roi et ses rapports avec son entourage? Notre information est ici encore plus mince: quelques pages dans un nombre limité de textes évoquant la vie quotidienne des monarques chez les Mossi, les Nupe, les Banyoro, les Banyankole; quelques notes plus importantes pour les Mamprusi, les Jukun, les Baganda, les Zulu. Un seul chapitre sur l'étiquette dans toute la littérature: il concerne les Kotoko; un seul traité de l'étiquette rédigé par les Africains: il concerne les Bamoum et fut établi à la demande de Njoya. Ces textes nous informent surtout des règles touchant la vie publique du souverain; nous ne savons presque rien de ses rapports avec ses épouses, peu de choses 
concernant sa personne, à l'exception de la façon dont il s'alimente ou dont il est parfois traité lorsqu'il est malade. Rien sur sa vie sexuelle; rien sur sa toilette.

Or, si je me fonde sur les informations que donne le texte bamoum sur la personne royale, elles montrent que celle-ci a deux faces, une publique et une privée. Ceci permet de mieux saisir, lorsqu'il s'agit du personnage public, la place faite dans l'étiquette à la rhétorique politique, au gestuel théâtral. Les observateurs européens ont maintes fois été abusés sur la nature du pouvoir des souverains africains par l'apparat et le style des cours royales: il suffit pour s'en convaincre de relire les textes des voyageurs du 19ème siècle.

Cette situation de l'information prise en considération, est-on tenu de comparer les figures des rois sacrés telles qu'elles se dégagent des mises en scène rituelles ou des métaphores de la rhétorique politique où foisonne un symbolisme qui, par sa nature même ou son jaillissement, ne se structure guère? Serait-ce nous éloigner du projet que de comparer les relations que les monarques entretiennent avec certains éléments de la société où il resterait légitime d'utiliser ce que les rituels mettent en évidence et ce que prescrivent les étiquettes?

On peut envisager quelques situations privilégiées:

a) Les relations du roi avec certains parents, mère et soeurs. Il y a là en effet des variations qui peuvent s'éclairer les unes par les autres: les mères de rois ne sont pas en effet toujours rapprochées de leur fils pour prendre place dans la façade politique, il y a au contraire des cas où elles sont écartées, où le roi ne doit plus les voir; c'est ce qui se passe chez les Jukun et les Igala (Meek, 1931: 137; Clifford, 1936: 420).

Les relations avec les soeurs posent des problèmes d'interprétation: l'inceste, réel ou symbolique, accompli avec des soeurs réelles ou classificatoires, doit-il s'interpréter comme l'affirmation que le roi n'a plus de parents et donc qu'il n'y a pas d'inceste ? Ou au contraire affirme-t-il, comme l'a proposé Luc de Heusch, que le roi est un transgresseur ? Il existe en effet dans les rites d'intronisation de nombreuses pratiques exprimant que le roi n'a plus de parents 
reconnus et qu'il jugera en conséquence sans privilégier les siens. L'inceste est-il un élément de cet engagement ou non ?

b) Les relations du roi avec les représentants de la population: les membres du lignage royal, les membres des appareils politiques - les faiseurs de roi -, les représentants des différentes composantes de la population, tels que les maîtres de la terre, les rois soumis. Il y a dans ce domaine une information riche et des rites faciles à saisir: on pense au périple du nouveau roi mossi, le ringu, et à ses équivalents ailleurs. Les comparaisons pourraient éclairer la place du roi, ses droits et ses obligations en face du "pays".

c) Il y aurait aussi les relations avec les ancêtres. Ceux-ci sont capitaux puisqu'ils seront propitiés directement ou indirectement et que leur bienveillance est tenue pour essentielle à l'heureuse conduite des affaires publiques.

Ce que l'on en sait à partir des intronisations est fort variable: les rois parfois absorbent une part du coeur de leur prédécesseur, entrent en contact avec le corps des morts, séjournent auprès des tombes, touchent des emblèmes. Que sont de plus ces remèdes si souvent absorbés lors des rituels ? Quelle est la signifcation des identifications avec les ancêtres ? Identité qui, remarquons-le, n'empêche pas que le monarque ne s'en distingue puisqu'il les prie et leur offre des sacrifices. Faits et idéologies ici restent mal cernés.

Répondons enfin à une dernière question qui nous est posée dans le libellé de l'invitation qui nous a été faite: le roi sacré est-il une machine de production illusoire ? Rappelons, en face d'une pareille interrogation, cette conclusion de Bloch sur les rois thaumaturges: "Ainsi, il est difficile de voir dans la foi au miracle royal autre chose qu'une erreur collective" (Bloch, 1983: 429). On pourrait, en plagiant l'historien, écrire qu'il est difficile de voir, dans la foi dans le pouvoir des rois africains, autre chose que l'erreur d'un continent.

L'anthropologue ne peut toutefois perdre de vue qu'il doit d'abord comprendre les fonctions et les cohérences internes des pratiques rituelles et des idéologies exprimées et saisir les problèmes que cellesci ont rencontrés au cours de l'histoire des sociétés étudiées. Evaluer 
les actes et les pensées des Africains en les rapprochant des représentations qui sont celles d'une pensée occidentale façonnée directement ou indirectement par la science n'est certes pas illégitime, mais ceci ne peut prévaloir contre l'obligation de comprendre d'abord les autres.

Claude Tardits

Ecole Pratique des Hautes Etudes

\section{Références bibliographiques}

Abrahams R.G.

1967 The Political Organization of Unyamwezi, Cambridge, Cambridge University Press.

Balandier G.

1969 Anthropologie politique, Paris, Albin Michel.

Bloch M.

1949 La société féodale. Les classes et le gouvernement des hommes. Paris, Albin Michel.

1983 Les rois thaumaturges: étude sur le caractère surnaturel at tribué à la puissance royale particulièrement en France et en Angleterre. Paris, Gallimard (1ère ed. 1924).

Bösch F.

1930 Les Bayamwezi: peuple de l'Afrique orientale, Münster, Anthropos.

Chilver E.M.

1959 "Feudalism in the Interlacustrin Kingdoms" in East African Chiefs, A. Richards Ed., London, Faber and Faber.

Clifford M.

1936 "A Nigerian Chiefdom. Some Notes on the Igala Tribes in Nigeria and their "Divine King", Journal of the Royal Anthropological Institute, 66.

Drucker Brown S.

1975 "Ritual Aspects of the Mamprusi Kingship" in African Social Research Document 6, Leiden, Afrika Studie Centrum. 
Dupire M.

1962 Peuls nomades, Paris, Institut d'Ethnologie.

Evans-Pritchard E. E.

1948 The Divine Kingship of the Shilluk of the Nilotic Sudan, Cambridge, Cambridge University Press.

Goody J .

1971 "Technology Tradition and the State" in Africa, Oxford, International African Institute and Oxford University Press.

Heusch L. de

1982 Rois nés d'un coeur de vache, Paris, Gallimard.

Izard M.

1985 Gens du pouvoir, gens de la terre. Les institutions politiques de l'ancien royaume du Yatenga (Bassin de la Volta Blanche), Paris, Maison des sciences de 1'Homme.

Jeffreys M.D.W.

1935 "The Divine Umundri King", Africa, VIII, 3.

Krige E.J.

1936 The Social System of the Zulu, Londres, Longmans.

Krige E.J. and J.D.

1943 The Realm of a Rain-Queen. A study of the Pattern of Lovedu Society, Londres, Oxford University Press.

Labouret $\mathrm{H}$.

1941 Paysans d'Afrique occidentale, Paris, Gallimard.

Lebeuf M.D.A.

1969 Les principautés kotoko. Essai sur le caractère sacré de l'autorité, Paris, CNRS.

Mair L.

1962 Primitive Government, Harmondsworth (Middlesex), Penguin Books.

Meek C.K.

1931 A Sudanese Kingdom: an Ethnological Study of the JukunSpeaking Peoples of Nigeria, Londres, Kegan Paul.

Meyerowitz E.L.R.

1960 The Divine Kingship in Ghana and Ancient Egypt, Londres, Faber and Faber.

Morton-Williams

1964 "An Outline of the Cosmology and the Cult Organization in the Oyo Yoruba", Africa, XXXIV, 3. 
Nadel S.F.

1942 A black Byzantium. The Kingdom of Nupe in Nigeria, Londres, Oxford University Press.

Njoya

1952 Histoire et coutumes des Bamum, rédigées sous la direction du Sultan Njoya. Traduction du Pasteur Henri Martin. (Mémoires de 1'IFAN, Centre du Cameroun).

Parrinder E.G.

1956 "Divine Kingship in West Africa", Numen, 3.

Richards A.I.

1968 The Henry Myers Lectures 1968. Keeping the King Divine, Journal of the Royal Anthropological Institute.

Richards A.I. ed.

1959 East African Chiefs. A Study of Political Development in some Uganda and Tanganyika Tribes. Londres, Faber and Faber, East African Institute of Social Research.

Roscoe J.

1911 The Baganda. An account of their Native Customs and Beliefs. Londres, MacMillan.

1923 The Bakitara or Banyoro. (The first part of the report of the Mackie ethnological expedition to Central Africa), Cambridge, Cambridge University Press.

1923 The Banyankole. (Second part of the report of the Mackie ethnological expedition. to Central Africa), Cambridge, Cambridge University Press.

Seligman C.G.

1934 Egypt and Negro Africa. A Study in Divine Kingship (Frazer lecture from 1933), Londres, Routledge and sons.

Tardits C.

1980 Le royaume bamoum, Paris, Peeters. 